REVIEW

\title{
Acute disseminated encephalomyelitis or multiple sclerosis: can the initial presentation help in establishing a correct diagnosis?
}

\author{
R C Dale, J A Branson
}

Arch Dis Child 2005;90:636-639. doi: 10.1136/adc.2004.062935

The differential diagnosis of CNS white matter disease is broad, and can be divided into vascular, metabolic, infective, or inflammatory aetiologies. Isolated inflammatory disorders of the CNS are often associated with demyelination, and the two terms (inflammatory and demyelinating) are often used in conjunction. When the disease is monophasic, the term acute disseminated encephalomyelitis (ADEM) is used. ' ADEM typically occurs as a post-infectious phenomenon, and by definition, must be an isolated (monophasic) episode. If a relapse occurs shortly after the ADEM presentation in association with a further infection or steroid withdrawal, the term MDEM (multiphasic disseminated encephalomyelitis) is used. When there are relapses or progressive disease, the term multiple sclerosis (MS) is used (for full recommended diagnostic criteria for multiple sclerosis refer to McDonald and colleagues ${ }^{2}$ ).

See end of article for authors' affiliations

Correspondence to: Dr R C Dale,

Neuroimmunology Laboratory, 9th Floor Institute of Neurology, Queen Square, London WCIN 3BG, UK; r.dale@ ion.ucl.ac.uk

Accepted 28 October 2004
$\mathrm{T}$ he diagnostic differentiation between acute disseminated encephalomyelitis (ADEM) and multiple sclerosis (MS) is important mainly for prognostic reasons. Children with ADEM are generally expected to do well, whereas children with MS are more likely to develop significant disability. The ability to reduce MS disease progression with immunomodulatory drugs further emphasises the importance of a prompt and accurate diagnosis. Over the last five years, there have been a number of large series reporting the clinical features of paediatric ADEM, MS, and the differences between these $^{3-8}$ A large follow up series of children with a first episode of central nervous system (CNS) inflammatory demyelination showed a much higher rate of progression to multiple sclerosis than previously reported. ${ }^{8}$ The clinical features of these treatable disorders are therefore the subject of this review. It must be stated from the outset that the clinical and laboratory differences between ADEM and MS are trends only, and do not provide rigid diagnostic criteria.

\section{THE DIAGNOSTIC DILEMMA}

The research purpose of this review is to address the diagnostic dilemma presented in fig 1. Are ADEM and MS distinct clinical disorders, or part of a disease spectrum? By definition, both ADEM and MS cases must manifest disseminated disease of the CNS (more than one clinical or radiological site). Diseases isolated to specific areas of the CNS (isolated optic neuritis, transverse myelitis, and brain stem dysfunction) are considered distinct from both ADEM and MS (clinically and prognostically), and will not be discussed in this review. ${ }^{8}$

\section{DEMOGRAPHICS}

Monophasic ADEM is more common in children, whereas MS is more common in adults. Between $2.7 \%$ and $4.4 \%$ of MS presentations occur in children less than 16 years of age. ${ }^{8}$ Mikaeloff et al showed a mean age of 7.1 years and 12.0 years in paediatric ADEM and MS patients respectively. ${ }^{8}$ Most reports of ADEM have described a peak incidence in children of 3-10 years. ${ }^{3-6}$ An adolescent presenting with a first demyelinating event is more likely to develop multiple sclerosis than a younger child. ${ }^{3}$ Most series of ADEM have failed to show a sex predominance, although some series show a mild male predominance. $^{358}$ By contrast, females are more predisposed to develop multiple sclerosis, particularly in adolescence and adulthood. ${ }^{8}$

\section{PRECIPITATING INFECTION AND SEASONALITY}

As would be expected from an infection mediated syndrome, ADEM most commonly presents during winter and spring. ${ }^{3}$ Between $51 \%$ and $74 \%$ of ADEM patients have a history of a precipitating infection with a mean latency of approximately two weeks. ${ }^{3-68}$ A large number of infections may precipitate ADEM, although isolation of a specific agent is uncommon (classic infectious precipitants include measles, EpsteinBarr virus, mycoplasma, and group A streptococcus). ${ }^{10}$ Although infections may precipitate an MS relapse, the association with infections and seasonality is less pronounced (only 16\% of patients have an infection in the preceding month $\left.{ }^{8}\right)$.

\section{NEUROLOGICAL SYMPTOMS AND SIGNS}

Patients with ADEM are more likely to present with encephalopathy and may be initially diagnosed as having viral encephalitis.. ${ }^{8}$ ADEM patients commonly have headache, vomiting,

Abbreviations: ADEM, acute disseminated encephalomyelitis; CNS, central nervous system; MDEM, multiphasic disseminated encephalomyelitis; MS, multiple sclerosis; ON, optic neuritis 


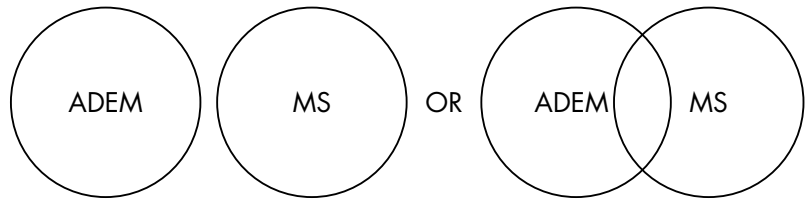

Figure 1 Diagnostic dilemma: are ADEM and MS separate clinical entities or part of the same demyelinating spectrum?

drowsiness, and meningism. These symptoms are uncommon in MS. ${ }^{368}$ Seizures occur in $13-35 \%$ of ADEM patients, although they are seldom as problematic as those seen in viral encephalitis, which is more likely to involve cortical grey matter. ${ }^{3-6}$ By contrast, seizures are considered rare in multiple sclerosis. ${ }^{11}$

As both ADEM and MS are disseminated disorders of the CNS, a broad range of neurological signs is possible. Pyramidal, cerebellar, and brain stem signs are common in both disorders. ${ }^{3}{ }^{8}$ Encephalopathy with depressed consciousness and altered sensorium is more common in ADEM (45$75 \%)^{3-68}$ than in MS (13-15\%). ${ }^{3}$ Optic neuritis (ON) occurs in both ADEM and MS. ON is frequently bilateral in ADEM, whereas it is typically unilateral in MS. ${ }^{13} 1213$ Morrissey's study also noted the importance of considering Leber's hereditary optic neuropathy (a mitochondrial mutation) in children presenting with bilateral optic neuritis/neuropathy. ${ }^{13}$ As ADEM tends to be more florid, there are frequently multiple symptoms and signs (polysymptomatic presentation), whereas symptoms and signs are commonly isolated (monosymptomatic) in MS. ${ }^{38}$

\section{BLOOD AND CSF FEATURES}

In keeping with an explosive post-infectious phenomenon, patients with ADEM frequently have raised inflammatory markers (white cell count, erythrocyte sedimentation rate) and lymphopenia. ${ }^{3}{ }^{4}$ In contrast, in MS these are frequently normal. ${ }^{3}$ The CSF more commonly shows an increased protein and cell count (lymphocytosis) in ADEM, although normal and abnormal CSF findings occur in both ADEM and MS. ${ }^{3-68}$ One of the most discriminating CSF findings is the presence of intrathecal synthesis of oligoclonal bands (oligoclonal IgG in CSF but not in serum), which occurs in
$40-95 \%$ of MS patients, but only $0-29 \%$ of ADEM patients. ${ }^{3568}$ Indeed, some authorities would seriously question the diagnosis of MS if there were not intrathecal synthesis of oligoclonal IgG.

\section{MAGNETIC RESONANCE NEUROIMAGING}

MRI is an essential part of the investigation of ADEM and MS. Both ADEM and MS show disseminated inflammatory lesions throughout the CNS (although predominantly in the white matter). Occasionally, large mass-like lesions occur and may require a biopsy. ${ }^{5614} \mathrm{~A}$ number of studies have reported imaging differences between childhood ADEM and MS. The lesions in ADEM often have poorly defined margins, ${ }^{3-6}{ }^{15}$ whereas MS lesions have well defined "plaquelike" margins. ${ }^{16}$ There are also differences in the lesion sites. Periaqueductal, corpus callosum, and periventricular white matter lesions are characteristic of $\mathrm{MS}^{3}{ }^{35}$ By contrast, in ADEM the lesions tend to be in the deeper white matter with periventricular sparing (only $29-60 \%$ of ADEM patients have periventricular lesions) (fig 2). ${ }^{346}$ When the spinal cord is involved in ADEM, the lesion is typically large, swollen, and thoracic. The spinal cord lesions in MS are typically smaller, more discrete, and cervical. In addition, although the white matter is classically involved in both disorders, the grey matter (both cortical and deep grey/basal ganglia) is frequently involved in ADEM (in contrast to MS)..$^{3-5} 151718$ Post-streptococcal ADEM shows particular predisposition to basal ganglia lesions. ${ }^{19}$ A recent MRI study of 116 children with a first episode of inflammatory demyelination showed that perpendicular corpus callosum lesions and the sole presence of well defined lesions were the most specific predictive factors for relapse (although they had a low sensitivity)..$^{20}$

Follow up MRI is useful in ADEM/MS differentiation. As would be expected, new lesions should not occur in ADEM (0-9\% of ADEM patients have new lesions on follow up). ${ }^{352122}$ The original lesions in ADEM completely resolve in $27-55 \%$ of ADEM patients, although more typically (45$64 \%$ ) the lesions only partially resolve. ${ }^{32021}$ By contrast, new lesions in MS are anticipated. ${ }^{17}$ The timing of repeat scanning is important, as too hasty a repeat scan may cause confusion if the patient is still in the acute/subacute phase. A lag time of six months after presentation would be appropriate for repeat scanning (when clinically indicated).
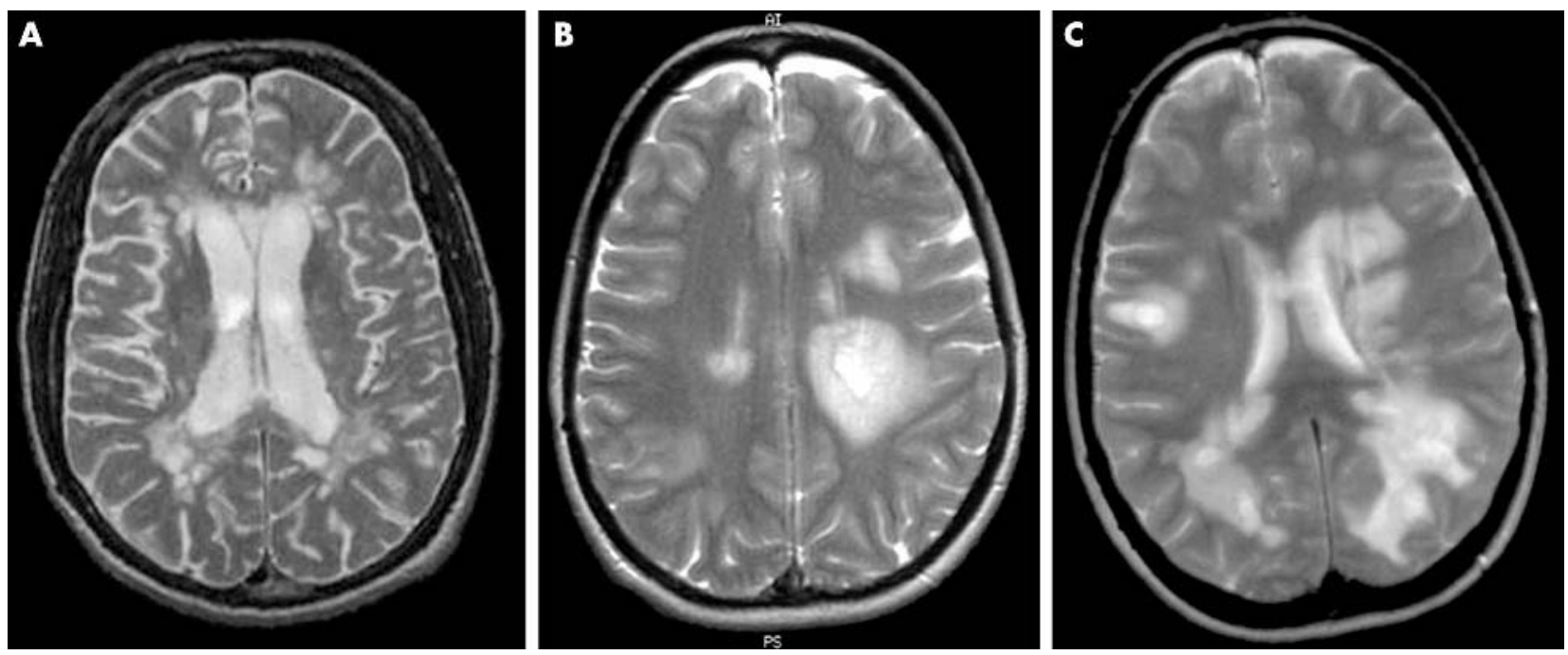

Figure 2 (A) MRI brain (T2 weighted) in MS showing well demarcated lesions in the region of the periventricular white matter. (B) MRI brain (T2 weighted) in ADEM showing a large mass-like lesion in the white matter. (C) MRI brain in ADEM showing multiple large lesions with poorly defined margins and relative periventricular sparing. 
ADEM

MS

$<10$ years

Present

Polysymptomatic

Bilateral

Cortical and deep

grey matter lesions

Lymphocytosis

No new lesions

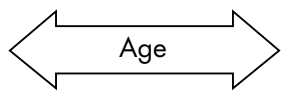

$>10$ years

Absent

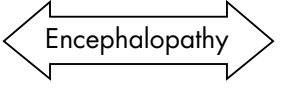

Monosymptomatic
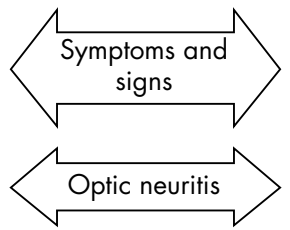

Unilateral

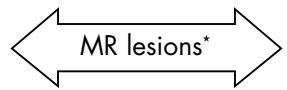

Periventricular/ callosal lesions

Intrathecal $\lg G$

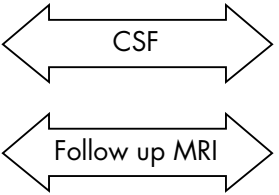

New lesions

Figure 3 Clinical and investigation differences between ADEM and MS (trends only). "MR lesions other than white matter.

\section{COMPARISON WITH ADULT STUDIES}

As MS in adults is common, the clinical, laboratory, and imaging features are well documented. By contrast, ADEM in adults is uncommon and frequently patients originally diagnosed with ADEM relapse and are reclassified as MS. The one study of ADEM in adults found that, of 40 patients, 14 progressed to MS after a mean of 38 months. ${ }^{23}$ The possibility that this adult ADEM cohort is still "contaminated" by MS is possible. ${ }^{18}$ However, the adult study did highlight similar differences between ADEM and MS to those described in paediatric cohorts: namely, preceding infection, fever, meningism, reduced consciousness, CSF lymphocytosis, and grey matter MR lesions were more common in ADEM. Intrathecal synthesis of oligoclonal bands, periventricular/callosal white matter lesions were more common in MS. ${ }^{23}$ However, the differences were less manifest in adults than in children, and were less likely to differentiate ADEM from MS at presentation. ${ }^{1823}$

\section{TREATMENT}

There have been no controlled trials to determine the efficacy of immunomodulatory treatments in childhood MS or ADEM. However, most patients with suspected inflammatory demyelinating CNS diseases are treated with steroids. It is common practice to use $10-30 \mathrm{mg} / \mathrm{kg} /$ day intravenous methylprednisolone (maximum $1 \mathrm{~g}$ ) for three days, although some clinicians use oral prednisolone, or even defer treatment if the patient is spontaneously improving. ${ }^{3-6}$ Studies have shown that a tapered course of oral prednisolone (over 2-6 weeks) reduces the chance of early onset relapse-that is, MDEM (although these studies were retrospective). ${ }^{322}$ Prophylactic immunomodulation to prevent relapses in MS has not been thoroughly tested in childhood MS. It is prudent for patients to remain on antivirals and antibiotics until viral/ bacterial encephalitis can be excluded..$^{510}$

\section{OUTCOME}

A recent important study of French children $(n=296)$ who suffered one episode of acute CNS inflammatory demyelination found a higher risk of progression to multiple sclerosis than previously reported (57\%). ${ }^{8}$ This study has highlighted the need for caution in counselling parents regarding the risk of further events.

The outcome in ADEM is often good with $57-81 \%$ of patients making a complete recovery. ${ }^{3-5} 21$ In children who have suffered one episode of inflammatory demyelination, adverse prognostic factors for irreversible disability include: sequelae after the first episode, a polysymptomatic presentation, progressive evolution, and the number of relapses in the first two years. ${ }^{8} 24$

\section{CONCLUSION}

The recent published reports of ADEM and MS in children have highlighted some differences between the two conditions, which are reviewed in fig 3. It must be reiterated that these features are trends only, and there remains no diagnostic test for multiple sclerosis. Despite the significant advances in our understanding of the pathogenesis of inflammatory demyelinating CNS disorders, the only truly reliable diagnostic test remains time.

\section{ACKNOWLEDGEMENTS}

RCD has a training fellowship awarded by Action Medical Research and the Barnwood House trust. We would like to thank Gavin Giovannoni for his support.

\section{Authors' affiliations}

R C Dale, J A Branson, Great Ormond Street Hospital NHS Trust and Institute of Child Health, London, UK

Competing interests: none declared

\section{REFERENCES}

1 Tselis AC, Lisak RP. Acute disseminated encephalomyelitis. In: Antel J, Birnbaum G, Hartung HP, eds. Clinical neuroimmunology. Blackwell Science, 1998:116-146.

2 McDonald WI, Compston A, Edan G, et al. Recommended diagnostic criteria for multiple sclerosis: guidelines from the International Panel on the diagnosis of multiple sclerosis. Ann Neurol 2001;50:121-7.

3 Dale RC, de Sousa C, Chong WK, et al. Acute disseminated encephalomyelitis, multiphasic disseminated encephalomyelitis and multiple sclerosis in children. Brain 2000;123(pt 12):2407-22.

4 Murthy SN, Faden HS, Cohen ME, et al. Acute disseminated encephalomyelitis in children. Pediatrics 2002;110(2 pt 1):e21.

5 Tenembaum S, Chamoles N, Fejerman N. Acute disseminated encephalomyelitis: a long-term follow-up study of 84 pediatric patients. Neurology 2002;59:1224-31.

6 Hynson JL, Kornberg AJ, Coleman LT, et al. Clinical and neuroradiologic features of acute disseminated encephalomyelitis in children. Neurology 2001;56:1308-12.

7 Gupte G, Stonehouse M, Wassmer E, et al. Acute disseminated encephalomyelitis: a review of 18 cases in childhood. J Paediatr Child Health 2003;39:336-42.

8 Mikaeloff Y, Suissa S, Vallee L, et al. First episode of acute CNS inflammatory demyelination in childhood: prognostic factors for multiple sclerosis and disability. J Pediatr 2004; 144:246-52.

9 Brex PA, Miszkiel KA, O'Riordan Jl, et al. Assessing the risk of early multiple sclerosis in patients with clinically isolated syndromes: the role of a follow up MRI. J Neurol Neurosurg Psychiatry $2001 ; 70: 390-3$.

10 Stonehouse M, Gupte G, Wassmer E, et al. Acute disseminated encephalomyelitis: recognition in the hands of general paediatricians. Arch Dis Child 2003;88:122-4.

11 Poser CM. The pathogenesis of multiple sclerosis: a commentary. Clin Neurol Neurosurg 2000;102:191-4.

12 Parkin PJ, Hierons R, McDonald WI. Bilateral optic neuritis. A long-term follow-up. Brain 1984;107(pt 3):951-64.

13 Morrissey SP, Borruat FX, Miller DH, et al. Bilateral simultaneous optic neuropathy in adults: clinical, imaging, serological, and genetic studies. $J$ Neurol Neurosurg Psychiatry 1995;58:70-4.

14 Kepes JJ. Large focal tumor-like demyelinating lesions of the brain: intermediate entity between multiple sclerosis and acute disseminated encephalomyelitis? A study of 31 patients. Ann Neurol 1993;33:18-27.

15 Singh S, Prabhakar S, Korah IP, et al. Acute disseminated encephalomyelitis and multiple sclerosis: magnetic resonance imaging differentiation. Australas Radiol 2000;44:404-11.

16 Paty DW, Oger JJ, Kastrukoff LF, et al. MRI in the diagnosis of MS: a prospective study with comparison of clinical evaluation, evoked potentials, oligoclonal banding, and CT. Neurology 1988;38:180-5. 
17 Kesselring J, Miller DH, Robb SA, et al. Acute disseminated encephalomyelitis. MRI findings and the distinction from multiple sclerosis. Brain 1990;113(pt 2):291-302.

18 Hartung HP, Grossman RI. ADEM: distinct disease or part of the MS spectrum? Neurology 2001;56:1257-60.

19 Dale RC, Church AJ, Cardoso F, et al. Poststreptococcal acute disseminated encephalomyelitis with basal ganglia involvement and auto-reactive antibasal ganglia antibodies. Ann Neurol 2001:50:588-95.

20 Mikaeloff $Y$, Adamsbaum C, Husson B, et al. MRI prognostic factors for relapse after acute CNS inflammatory demyelination in childhood. Brain 2004; 127:1942-7.
21 O'Riordan JI, Gomez-Anson B, Moseley IF, et al. Long term MRI follow-up of patients with post infectious encephalomyelitis: evidence for a monophasic disease. J Neurol Sci 1999;167:132-6.

22 Anlar B, Basaran C, Kose G, et al. Acute disseminated encephalomyelitis in children: outcome and prognosis. Neuropediatrics 2003;34:194-9.

23 Schwarz S, Mohr A, Knauth M, et al. Acute disseminated encephalomyelitis: a follow-up study of 40 adult patients. Neurology 2001;56:1313-18.

24 Boiko A, Vorobeychik G, Paty D, et al. Early onset multiple sclerosis: a longitudinal study. Neurology 2002;59:1006-10.

25 Simone IL, Carrara D, Tortorella C, et al. Course and prognosis in early-onset MS: comparison with adult-onset forms. Neurology 2002;59:1922-8.

ARCHIVIST.

\section{German etanercept registry for JIA patients}

$\mathrm{E}$ tanercept, an anti-tumour necrosis factor $\alpha$ drug, has been approved and licensed for the treatment of active, treatment resistance, polyarticular juvenile idiopathic arthritis (JIA) in patients aged 4 years or older. An etanercept registry was begun in January 2001 by paediatric rheumatologists in Germany and Austria, and data from the first 34 months of the registry have been reported (G Horneff and colleagues. Annals of the Rheumatic Diseases 2004;63:1638-44).

Up to the end of October 2003 data had been collected on 322 patients from 36 centres. All had failed to respond to methotrexate before starting etanercept and they had been treated with etanercept for up to 48 months. Half of the patients had either systemic onset JIA $(21 \%)$ or seronegative polyarticular JIA $(29 \%)$. Twelve per cent had seropositive polyarticular JIA and $17 \%$ extended oligoarticular JIA. The remaining $21 \%$ had persistent oligoarticular JIA, enthesitis and arthritis JIA subtype, psoriasis and arthritis JIA subtype, or unclassified JIA. Patients with systemic onset JIA had more severe disease. A therapeutic effect of etanercept was documented at 1 month and increased throughout the first year. Overall, a $70 \%$ improvement was achieved in $30 \%$ at 1 month and $54 \%$ at 12 months. A $50 \%$ improvement was achieved in 54\% at 1 month and $71 \%$ at 12 months. Among patients with systemic onset JIA the $70 \%$ improvement rates were $11 \%$ at 1 month and around $30 \%$ at 12 months, and the corresponding 50\% improvement rates were 33\% and 39\%. Among 66 patients with systemic onset JIA $14(21 \%)$ discontinued etanercept because of lack of effectiveness. Among 256 patients with non-systemic disease 11 (4\%) stopped treatment because it was ineffective. Complete remission occurred in $26 \%$ of patients overall and in $13 \%$ of those with systemic onset disease. Treatment was stopped because of disease remission in 14 patients. Six of these had a relapse after $1-11$ months. Re-treatment in five patients was successful. Sixty-nine adverse events were reported in 56 patients. Eleven patients stopped treatment because of an adverse event-uveitis in three patients. Twelve adverse events were judged to be severe. The most common adverse events were local skin reaction (7), raised liver enzymes (7), and itching and a rash (6). Twenty patients had a variety of infections, the most serious of which was pneumonia, requiring mechanical ventilation. One patient developed central nervous system demyelination. Demyelination has been reported in three patients with JIA, and 17 with other forms of arthritis, on treatment with either etanercept or infliximab. The authors of this paper suggest performing MRI scans of the central nervous system in selected patients.

Etanercept is effective treatment for patients with refractory JIA. Improvement occurs within the first month of treatment and the rate and degree of improvement increase throughout the first year. Patients with systemic onset disease respond less well. The treatment is well tolerated on the whole. 